\title{
Development of Active Rocking Chair with Changing Swing According to Heartbeat Fluctuation*
}

\author{
Takeshi KAWASHIMA**
}

\begin{abstract}
The purpose of this study is to realize a comfortable swing according to the condition of each individual person. A simple active rocking chair is developed, the swing of which changes with the heartbeat fluctuation of the seated person. The active rocking chair is driven by a solenoid for safety and silence. Swinging is strengthened when the period of the heartbeat lengthens, and it is weakened when the period shortens. Swinging is evaluated using a questionnaire of 16 bipolar scales that was developed for the evaluation of comfortable swinging on the basis of the semantic differential (SD) technique. As a result, it is confirmed that comfortable swinging is realized by the developed active rocking chair. Then the acceleration near the ear of the seated person is measured in order to examine the features of comfortable swinging using the feedback of heartbeat fluctuation. It is clarified that the power spectrum of the envelope of the acceleration waveform shows " $1 / f$ fluctuation".
\end{abstract}

Key Words: Environmental Engineering, Human Interface, Human Engineering, Comfortable Swing, Heartbeat, Fluctuation, Rocking Chair, Active Swing, SD Technique, $1 / f$ Fluctuation

\section{Introduction}

It is possible to classify sound into "noise" and "music." The "noise" causes a person to become stressed, and the "music" makes a person feel relaxed. Although vibration is a phenomenon similar to sound, "vibration" which makes a person feel relaxed is difficult to imagine. The joggle of a train fundamentally deteriorates the riding comfort, but it also is the stimulation that induces comfortable sleep. That is to say, vibration may have both adverse and favorable properties, similarly to sound. The improvement of both the frequency characteristics and the tone quality by waveform shaping is effective for noise reduction $^{(1)-(4)}$. Therefore, it may also be effective for vibration control. In order to realize this, it is necessary to clarify the features of the vibration with which a person becomes comfortable. Therefore, the author clarified one pattern of swinging with which a person became comfortable: swinging where " $1 / f$ fluctuation" is applied on the

* Received 29th March, 2006 (No. 04-1063). Japanese Original: Trans. Jpn. Soc. Mech. Eng., Vol.71, No.709, C (2005), pp.2731-2737 (Received 27th September, 2004)

** Department of System Design Engineering, Kanagawa Institute of Technology, 1030 Shimo-ogino, Atsugi-shi, Kanagawa 243-0292, Japan.

E-mail: kawasima@sd.kanagawa-it.ac.jp envelope. In addition, an active swing chair and an active rocking chair have been developed to realize comfortable swinging ${ }^{(5)}$. In this study, a simple active rocking chair, which offers comfortable swinging be fitting the condition of each individual person, is developed to create more comfortable swinging and to clarify its characteristics.

Feedback of biological signals is required to realize comfortable swinging according to the condition of each individual person. As a biological signal in which information on comfort is included, brain waves are considered. However, comfort is deteriorated by squeezing of the head when the headband for detecting the brain waves is put on. Instead, a clip-type heartbeat sensor often found on aerobic exercise machines is used, because the sensor is attached to the body but has minimal effect on sensation. The sensor is attached to the earlobe, and detects the heartbeat as a transmission quantity of infrared rays. Life support activity which adjusts internal conditions to the external environment is supported by the autonomic nervous system. The heart is also controlled by the autonomic nervous system. Therefore, though the quantity of information on the heartbeat is less than that obtained from brain waves and there is also a time delay, it is considered that the heartbeat will provide information on comfort, particularly information concerning uncomfortable swinging. In this study, the relationship between heartbeat and 
strength of swinging is examined using the swinging pattern obtained for comfortable swinging. Next, based on the result, an active rocking chair which realizes comfortable swinging according to the condition of each individual person is developed. Then, the performance of this chair is confirmed on the basis of a questionnaire of 16 bipolar scales of 5 points that was developed for the evaluation of comfortable swinging by the semantic differential (SD) technique. Finally, features of swinging of the active rocking chair using the feedback of biological signal are examined.

In a series of this research, "comfortable swinging" has been defined as "swinging which becomes the feeling as relaxing," because the definition of "comfortableness" is different by the individual.

\section{Heartbeat Fluctuation}

\subsection{Heartbeat fluctuation}

The heart pumps blood throughout the body. This action can be observed externally as the pulse and is characterized by blood pressure and period of the heartbeat. The period always fluctuates slightly, and this change is called "the heartbeat fluctuation". When analyzing the heartbeat fluctuation by fast Fourier transform, three peaks appear in the power spectrum. Therefore, the power spectrum can be divided into three frequency domains: $0.05 \mathrm{~Hz}$ or less (low-frequency range), $0.05 \sim 0.15 \mathrm{~Hz}$ (intermediate-frequency range), and $0.25 \sim 0.4 \mathrm{~Hz}$ (highfrequency range). It is reported that the fluctuation in the low-frequency range is related to the thermoregulation mechanism, the fluctuation in the intermediate-frequency range is related to the vasomotor activity, and the fluctuation in the high-frequency range is related to the respiratory movement ${ }^{(6)-(8)}$. In this study, comfortable swinging is realized by using this heartbeat fluctuation.

\subsection{Measurement of heartbeat fluctuation}

The heartbeat fluctuation can be calculated as the difference in the period of the pulse from the previous one. Therefore, the measuring system is composed of a cliptype ear sensor, a heartbeat detector and a microcomputer. Figure 1 shows the ear sensor and the heartbeat detector.

With the ear sensor, the earlobe is held between an infrared LED and a phototransistor, and it detects the blood flow as infrared-ray transmission. This signal is composed of direct current and a weak alternating current of a triangular waveform with a steep rising slope and a gentle downward slope. In the heartbeat detector, the signal from the ear sensor is converted to a TTL signal following the flowchart shown in Fig. 2. The direct current is first removed by AC coupling, and the weak alternating current is amplified 1470 times by the operational amplifier (LMC662). Then, the rising slope of the signal is detected by the differentiator and subsequently shaped into the TTL signal by the comparator. In the computer, the

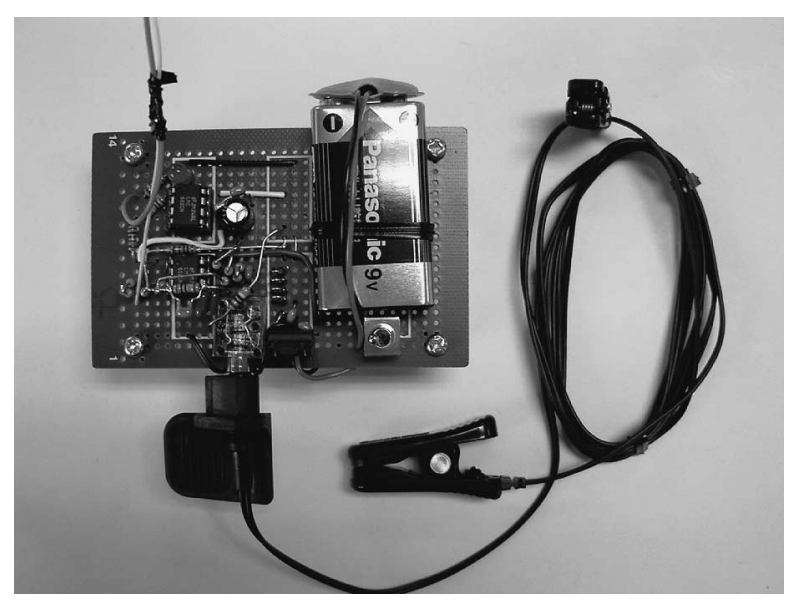

Fig. 1 Photograph of ear sensor and heartbeat detector

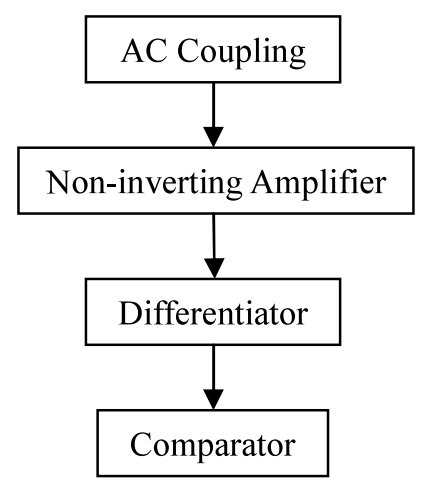

Fig. 2 Flowchart of heartbeat detector

period of the TTL signal is measured with a built-in timer $(3.125 \mathrm{MHz})$, and the heartbeat fluctuation is calculated from the difference between consecutive periods.

\section{Relationship between Heartbeat Fluctuation and Stimulus Intensity}

In this section, we clarify how to feed back the heartbeat fluctuation in order to realize the comfortable swinging be fitting the condition of each individual person. Then, the relationship between heartbeat fluctuation and strength of swinging at which a person becomes comfortable is examined by changing the fundamental frequency of the waveform for comfortable swinging.

\subsection{Waveform for comfortable swinging ${ }^{(5)}$}

Figure 3 shows the waveform, the swinging of which has been evaluated as being comfortable. The fundamental wave simulates the natural motion of the rocking chair. The frequency of the fundamental wave is $0.20 \mathrm{~Hz}$. The amplitude is modulated by the wave with an inverse relation between the power spectrum and the frequency (that is, " $1 / f$ fluctuation") in the frequency range of $0.0005 \sim 0.05 \mathrm{~Hz}$. The fundamental frequency of $0.20 \mathrm{~Hz}$ is chosen from the frequency range of $0.2 \sim 0.3 \mathrm{~Hz}$, at which swinging feels the most natural when the rocking chair is driven by a fixed-amplitude waveform of various 


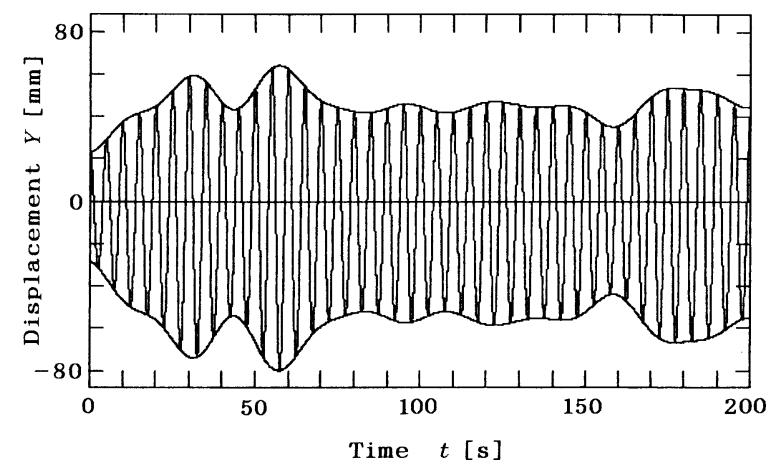

Fig. 3 Waveform for comfortable swinging

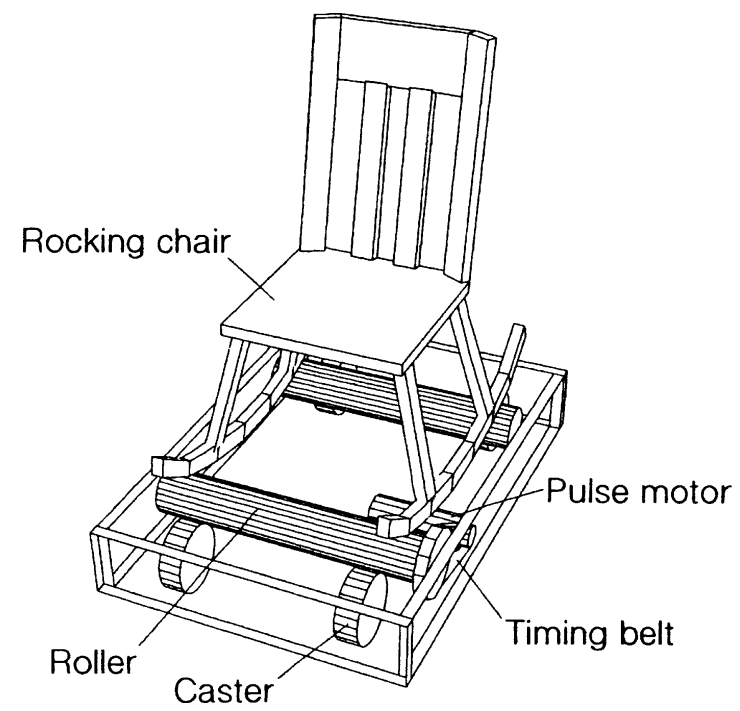

Fig. 4 Rocking chair driving apparatus

frequencies. The waveform modulating the amplitude is formed by superimposing 101 sinusoidal waves with an inverse relation between the square of amplitude and the frequency.

This swinging is realized by the drive unit shown in Fig. 4. This equipment can reproduce various types of swinging of the rocking chair.

\subsection{Relationship between heartbeat period and comfortable frequency}

The relationship between heartbeat period and comfortable fundamental frequency, that is to say, stimulus intensity, was examined in our laboratory under quiet conditions with the cooperation of 18 male and 2 female students 21 25 years old. Each examinee was explained the experimental procedure and the ear sensor was clipped onto the earlobe. Then, the examinee was subjected to swinging of the fundamental frequency of $0.24 \mathrm{~Hz}$ for 5 minutes, after which the examinee was requested to answer questions about the fundamental frequency. Depending on the answer, the fundamental frequency was changed to $0.20 \mathrm{~Hz}, 0.24 \mathrm{~Hz}$ or $0.28 \mathrm{~Hz}$. The new swinging mode was applied again for 5 minutes and evaluated. These procedures were repeated 3 times. Finally, the ex-
Table 1 Fundamental frequency evaluated as comfortable and period of heartbeat

\begin{tabular}{|c|c|c|c|c|}
\hline \multirow{2}{*}{ No. } & \multicolumn{5}{|c|}{ Frequency of fundamental wave [Hz]/ } \\
\cline { 2 - 5 } & $1^{\text {st }}$ trial & 2nd trial & 3rd trial & Final \\
\hline 1 & $0.24 / 0.68$ & $0.20 / 0.68$ & $0.20 / 0.68$ & $0.20 / 0.68$ \\
\hline 2 & $0.24 / 0.72$ & $0.20 / 0.71$ & $0.16 / 0.71$ & $0.20 / 0.71$ \\
\hline 3 & $0.24 / 0.73$ & $0.20 / 0.72$ & $0.24 / 0.72$ & $0.24 / 0.73$ \\
\hline 4 & $0.24 / 0.81$ & $0.24 / 0.85$ & $0.24 / 0.88$ & $0.24 / 0.84$ \\
\hline 5 & $0.24 / 0.98$ & $0.28 / 0.96$ & $0.28 / 0.95$ & $0.28 / 0.96$ \\
\hline 6 & $0.24 / 0.66$ & $0.28 / 0.64$ & $0.24 / 0.64$ & $0.24 / 0.65$ \\
\hline 7 & $0.24 / 0.84$ & $0.28 / 0.83$ & $0.28 / 0.84$ & $0.28 / 0.84$ \\
\hline 8 & $0.24 / 0.79$ & $0.20 / 0.85$ & $0.20 / 0.81$ & $0.20 / 0.82$ \\
\hline 9 & $0.24 / 0.86$ & $0.20 / 0.84$ & $0.16 / 0.88$ & $0.16 / 0.86$ \\
\hline 10 & $0.24 / 0.75$ & $0.28 / 0.76$ & $0.32 / 0.78$ & $0.28 / 0.76$ \\
\hline 11 & $0.24 / 0.63$ & $0.20 / 0.66$ & $0.16 / 0.68$ & $0.16 / 0.66$ \\
\hline 12 & $0.24 / 0.84$ & $0.28 / 0.84$ & $0.24 / 0.82$ & $0.28 / 0.84$ \\
\hline 13 & $0.24 / 0.96$ & $0.28 / 0.94$ & $0.32 / 0.94$ & $0.32 / 0.95$ \\
\hline 14 & $0.24 / 0.75$ & $0.28 / 0.76$ & $0.20 / 0.77$ & $0.20 / 0.76$ \\
\hline 15 & $0.24 / 0.86$ & $0.28 / 0.86$ & $0.28 / 0.86$ & $0.28 / 0.86$ \\
\hline 16 & $0.24 / 0.88$ & $0.28 / 0.86$ & $0.28 / 0.85$ & $0.28 / 0.86$ \\
\hline 17 & $0.24 / 0.54$ & $0.24 / 0.56$ & $0.20 / 0.58$ & $0.20 / 0.56$ \\
\hline 18 & $0.24 / 0.80$ & $0.24 / 0.80$ & $0.20 / 0.83$ & $0.20 / 0.81$ \\
\hline 19 & $0.24 / 0.77$ & $0.20 / 0.79$ & $0.20 / 0.84$ & $0.20 / 0.80$ \\
\hline 20 & $0.24 / 0.76$ & $0.20 / 0.82$ & $0.20 / 0.82$ & $0.20 / 0.80$ \\
\hline
\end{tabular}

aminee was requested to select the fundamental frequency, among $0.16 \mathrm{~Hz}, 0.20 \mathrm{~Hz}, 0.24 \mathrm{~Hz}, 0.28 \mathrm{~Hz}$ and $0.32 \mathrm{~Hz}$, at which swinging was the most comfortable. During swinging at each frequency, the period of the heartbeat was measured for the last 3 minutes, considering the adaptation time to each swinging frequency. The results are shown in Table 1. From this table, the following points can be confirmed.

(1) Swinging of the 2nd trial is not always more comfortable than one of 1 st trial. That is to say, there are 3 examples of returning to the original frequency in the 2nd judgment and 1 example of changing the frequency reverse to the 1 st judgment.

(2) In 6 out of 8 examples in which the average heartbeat period is over $0.83 \mathrm{~s}$, swinging with the fundamental frequency above $0.28 \mathrm{~Hz}$, that is to say, strong swinging, was finally evaluated as being comfortable.

( 3 ) In 9 out of 12 examples in which the average heartbeat period is less than $0.83 \mathrm{~s}$, swinging with the fundamental frequency below $0.20 \mathrm{~Hz}$ was evaluated as being comfortable. In 2 examples, swinging with the fundamental frequency of $0.24 \mathrm{~Hz}$ was judged to be comfortable. That is to say, in 11 out of 12 examples, strong swinging was finally judged to be uncomfortable.

The above results clarify that swinging should be driven by strong stimulation when the average heartbeat period is longer than $0.83 \mathrm{~s}$ and by weak stimulation when 


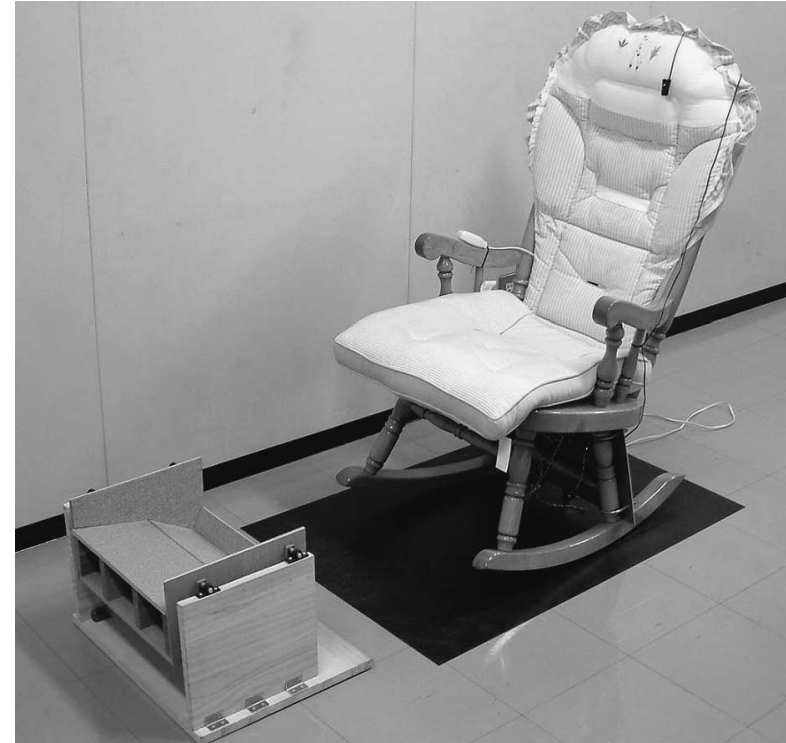

Fig. 5 Photograph of active rocking chair

the period is shorter than $0.83 \mathrm{~s}$ in order to realize comfortable swinging.

\section{Active Rocking Chair}

As a simple device to offer comfortable swinging be fitting the condition of each individual person, an active rocking chair feeding back the heartbeat fluctuation to the swing is developed, as shown in Fig. 5.

\section{1 Drive system}

A schematic drawing of active rocking chair is shown in Fig. 6. A commercially available wooden rocking chair is used. And a solenoid is selected for the drive, because it is desirable that the drive system be a safe and simple mechanism. The merits of the solenoid are low cost and light weight, but the driving force of the solenoid is difficult to control in comparison with the force of an electromagnetic cylinder. In addition, the rocking chair driven by the solenoid can be stopped safety by human force, and the drive is quiet because the solenoid has no contact point. The device can also be used as an ordinary rocking chair when the solenoid does not operate.

The rocking chair is driven by the solenoid pushing out the iron rod with the caster at the tip. The solenoid is controlled by a solid-state relay (SSR) that turns the current on and off via a control signal from the microcomputer. An ottoman (footstool), the footboard of which hangs on 4 arms from the base and moves freely, is prepared in order to reflect the change of solenoid force to swinging of the rocking chair. A photograph of the drive unit is shown in Fig. 7. The solenoid is installed at the center of the aluminum plate fixed to the rocking chair, the driving circuit using the solid-state relay is on the right side of the plate and the DC power source is on the left side.

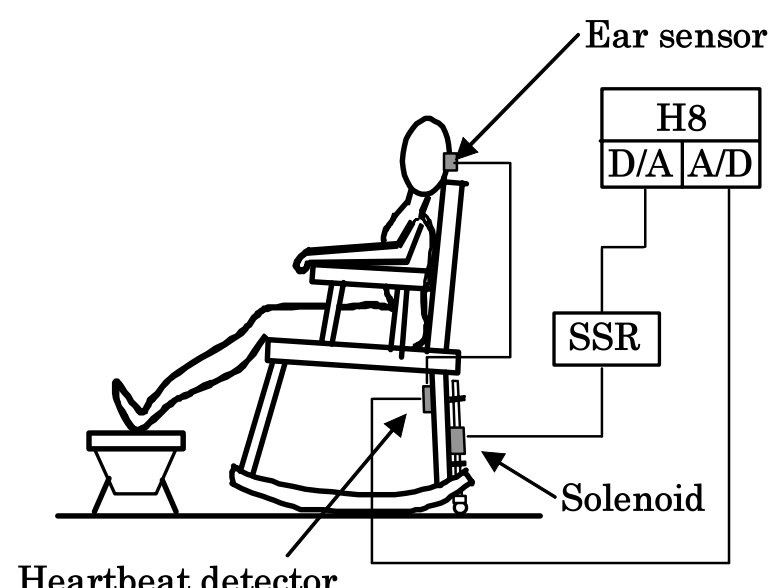

Fig. 6 Scheme of active rocking chair

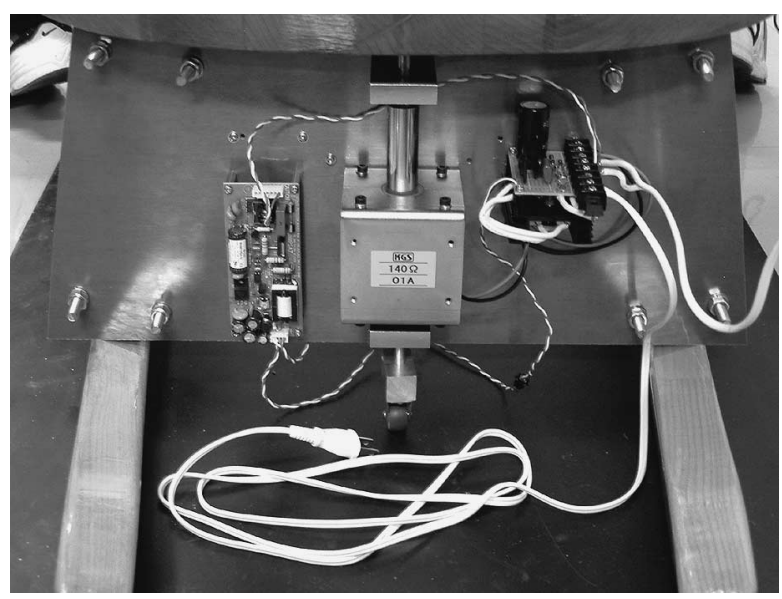

Fig. 7 Drive unit of active rocking chair

A headrest is necessary to support the neck when the rocking chair is driven, because the neck may not be strained to rock the chair. Therefore, the pillow commonly used in the bath is attached as a headrest at the upper part of the backrest. In addition, wooden seat and backrest feel hard when the chair is driven, because the body may not be strained to rock the chair. Therefore, a cushion is placed on the seat and backrest, as shown in Fig. 5.

\subsection{Control system}

The signal from a small gyroscope (MURATA MFG. Co.: ENV-05F-03) installed in the rocking chair is send to a one-board microcomputer (Akizuki Denshi Co.: H8$3052 \mathrm{~F}$ ) through a built-in AD converter, and the control signal is output through a built-in DA converter when the rocking chair moves forward, so that the solenoid operates in order to excite the self-induced oscillation. At the same time, in the one-board microcomputer, the operating time of the solenoid is determined on the basis of the heartbeat fluctuation calculated using the signal from the ear sensor.

The photograph of the control system is shown in Fig. 8. A circuit board with the microcomputer (center) and the heartbeat detector (right) are installed on the back 


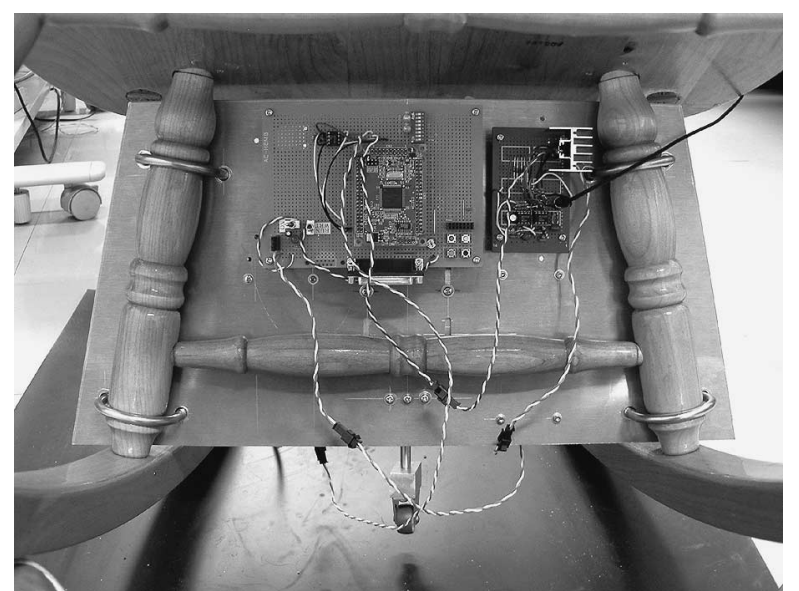

Fig. 8 Control unit of active rocking chair

of the plate in order to lessen the noise from the solenoid and the power supply.

\subsection{Driving algorithm}

In this study, an attempt was also made to very the swinging of the rocking chair by changing the solenoid force. However, swinging often stopped due to weak force, and the change of the driving force was not sensed by the examinee. Therefore, the fluctuation of swinging was induced by changing the operating time with constant solenoid force. This way, the change of swinging could be sensed by most of the examinees.

It has been confirmed that the stimulation should be changed according to the average heartbeat period. Therefore, in this study, the stimulation is strengthened when heartbeat period lengthens, and weakened when the period shortens. That is to say, as the driving algorithm, it is proposed that the operating time $T_{s}$ be a linear function of the heartbeat fluctuation $\Delta T$, as follows:

$$
T_{s}=0.6+\frac{31.25 \times 10^{3}}{4096} \Delta T .
$$

Each coefficient in Eq. (1) was obtained by trial and error in order that the examinee could sense the change of swinging. In addition, the lower limit of the operating time was set to $0.4 \mathrm{~s}$ so as not to stop swinging, and the upper limit was set to $0.8 \mathrm{~s}$, which was about the half of the natural period of the rocking chair of $1.8 \mathrm{~s}$, so that the solenoid operated only when the rocking chair moved forward.

\section{Evaluation Experiment}

The evaluation experiment was carried out to confirm the comfort of the developed active rocking chair.

\subsection{Evaluation method}

Swinging is evaluated using a questionnaire of 16 bipolar scales of 5 points that was developed for the evaluation of comfortable swinging. In this questionnaire, 16 crossbars with five scales are placed at regularly intervals, and epithet pairs, the epithet of which means the contrariety to each other, are typed at each end of the crossbars.

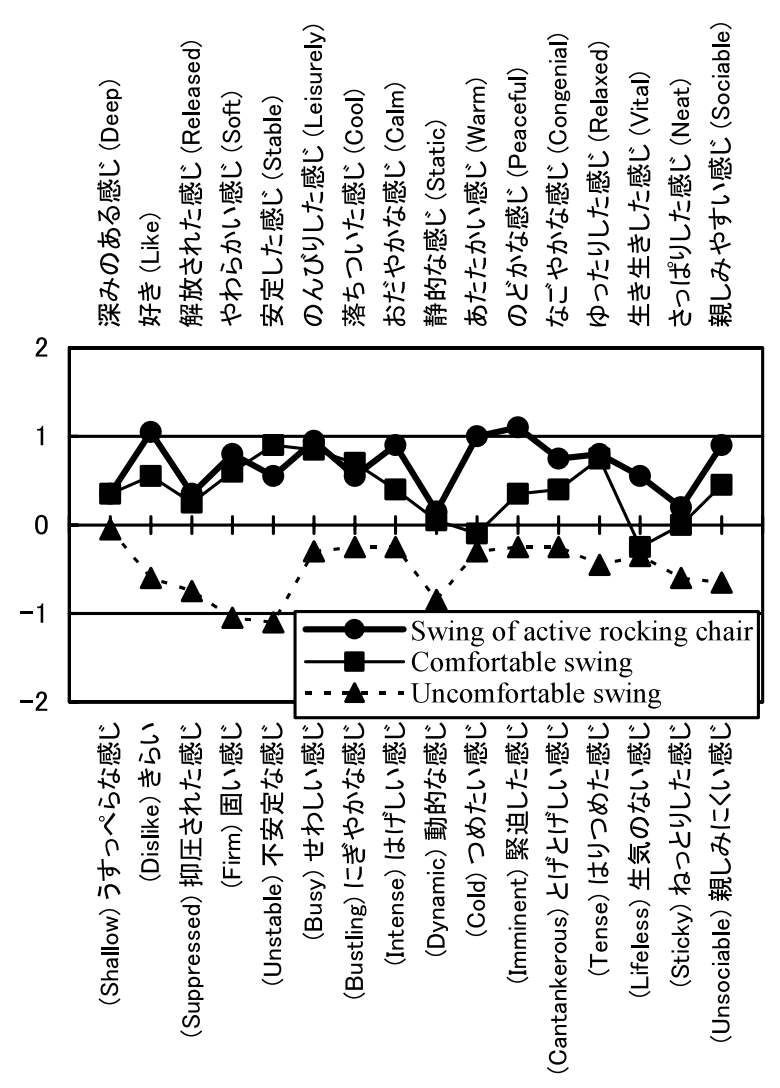

Fig. 9 Results of questionnaire (Averages)

Then, $\bigcirc$ is written by the examinee on the scale at the degree that is being most true for each epithet pair as an impression of swinging. This questionnaire was developed by factor analysis in the SD technique as follows. First, it was clarified that 49 epithet pairs were divided into 3 factors. Sixteen epithet pairs, which were comprehensible to the students, were selected from among the pairs corresponding to high load factor. Then, these epithet pairs were randomly listed in the questionnaire.

\subsection{Experimental method}

The evaluation experiment was carried out in our laboratory under quiet conditions with the cooperation of 20 male students. Each examinee was explained the experimental procedure and the ear sensor was clipped onto the earlobe. Then the examinee sat for a short while in the active rocking chair to become accustomed to it, and then was subjected to swinging for 10 minutes. Finally, the examinee was requested to fill in the questionnaire.

\subsection{Results and considerations}

The results of the evaluation experiment are shown in Fig. 9. The thick solid line shows the average value of swinging of the active rocking chair. In addition, the average value of swinging evaluated as being comfortable is shown as the thin solid line, and the average value of swinging evaluated as being uncomfortable is shown as the thin dotted line.

The evaluation values of swinging of the active rock- 


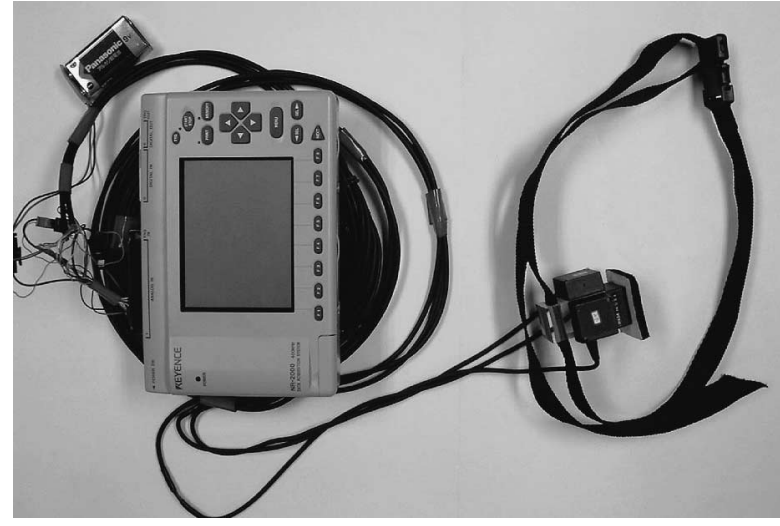

Fig. 10 Photograph of measurement system

ing chair are almost equal to or greater than the values of swinging evaluated as being comfortable. Therefore, it is confirmed that swinging of the developed active rocking chair is as comfortable as or more comfortable than swinging evaluated as being comfortable. In particular, high evaluations are obtained for the epithet pairs of "Vital - Lifeless" and "Like - Dislike." These favorable evaluations are a result of swinging being adapted to the condition of each individual person by feeding back bio-information of the examinee to the swing. In addition, high evaluations are obtained for the epithet pairs of "Warm - Cold" and "Peaceful - Imminent," as a result of installing a cushion on the seat and backrest of developed active rocking chair.

\section{Features of Swinging Changed According to Heartbeat}

The acceleration near the ear was measured in order to clarify the features of comfortable swinging changed according to the heartbeat fluctuation.

\subsection{Measurement system}

The semicircular canals in the internal ear of the human detect the rotation and the accelerated motion. Therefore, swinging and low-frequency vibration are sensed in the semicircular canals. In this study, the acceleration near the ear is measured to represent the acceleration in the semicircular canals.

The measurement system shown in Fig. 10 is composed of three small amplifier-equipped semiconductor acceleration transducers (Kyowa Electronic Instruments Co.: ASV-2GA, $-2 \mathrm{G}$ to $+2 \mathrm{G}$ ) and a battery-driven data recorder (Keyence: NR-2000, Size: $166 \times 110 \times 39.8$ ) so that it does not restrict the movement of the active rocking chair. The three acceleration transducers are attached on different planes of an acrylic rectangular parallelepiped block in order to measure three-axis acceleration, and these are driven by a $9 \mathrm{~V}$ dry battery (6LR61). The block is fixed near the ear of the examinee with the fabric belt, as shown in Fig. 11.

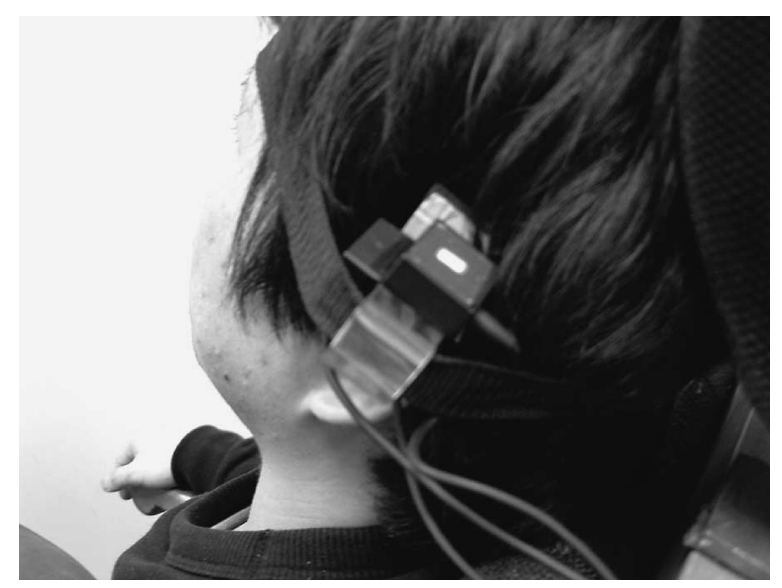

Fig. 11 Headband with three acceleration transducers

\subsection{Analysis of acceleration wave}

Because the power spectrum of the envelope of the waveform of swinging evaluated as being comfortable shows a relationship of " $1 / f$ fluctuation," the envelope of the measured acceleration wave is analyzed by frequency analysis. First, the peaks of the acceleration wave are detected, and regular-interval data of the envelope is obtained by interpolation. Then, the power spectrum of the envelope is obtained by fast Fourier transform. It is possible for the envelope of the displacement waveform and the envelope of the acceleration waveform to be considered almost equal, if the frequency of the envelope is much lower than the fundamental frequency of the wave.

\subsection{Measuring method}

The evaluation experiment was carried out in our laboratory under quiet conditions with the cooperation of 23 male students. The block with the three acceleration transducers was positioned near the ear of the examinee with the fabric belt, and swinging was carried out for 20 minutes. The acceleration near the ear was measured at the sampling frequency of $20 \mathrm{~Hz}$. Although the examinee could not be feeling comfortable by swinging because of the tight headband, he was made to experience naturally the swing.

\subsection{Power spectrum of envelope of acceleration wave}

The power spectra of each of the 23 examinees are shown in Fig. 12 in the unit of the measurement voltage, and the average power spectrum is shown in Fig. 13 in the unit of the acceleration. In addition, the relationship of $1 / f$ is shown by the broken line. In Fig. 12, the power spectra of all examinees show similar tendencies, but the magnitudes are different. In Fig. 13, the approximated slope of the average power spectrum is -0.98 in the frequency range of $0.001 \sim 0.1 \mathrm{~Hz}$, that is to say, the power spectrum of the envelope shows the relationship of " $1 / f$ fluctuation." Therefore, it is clarified that the power spectrum for swinging changed according to the heartbeat fluc- 


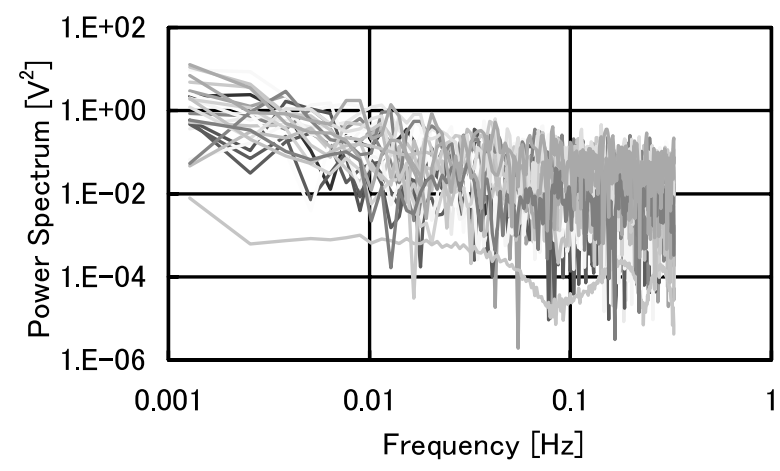

Fig. 12 Power spectra for each examinee

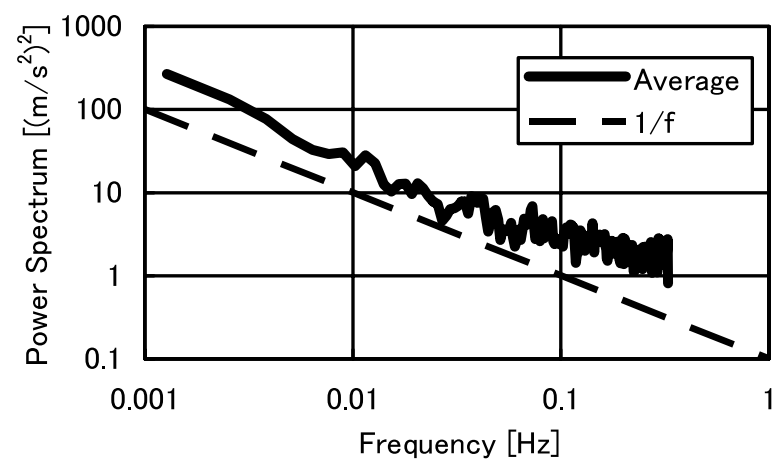

Fig. 13 Average power spectrum

tuation equals that for swinging evaluated as being comfortable. In other words, "comfortable swinging" can be realized merely by feeding back the heartbeat fluctuation to the swing.

\section{Conclusions}

( 1 ) In this study, a simple active rocking chair driven by a solenoid was developed to realize comfortable swinging be fitting the condition of each individual person by feeding back the heartbeat fluctuation to swinging of the active rocking chair. A driving algorithm in which the operating time is changed as a linear function of the heartbeat fluctuation is proposed; that is to say, the stimulation is strengthened when the heartbeat period lengthens and weakened when the period shortens.
(2) Swinging was evaluated using a questionnaire of 16 bipolar scales of 5 points that was developed for the evaluation of comfortable swinging. As the result, it was confirmed that comfortable swinging is realized by the developed active rocking chair which feeds back the heartbeat fluctuation of the rocked person to the swing.

( 3 ) The acceleration near the ear of the seated person was measured to examine the features of swinging with the heartbeat fluctuation feedback. It was clarified that the power spectrum of the envelope of the acceleration waveform shows the relationship of approximately $1 / f$, which generally appears in comfortable phenomena.

\section{References}

( 1 ) Hashimoto, T., Improvement of Sound Quality of Machinery Noise, Proc. 7th Symposium on Environmental Engineering, (in Japanese), No.97-2 (1997), pp.75-80.

(2) Hashimoto, T., Procedure for the Improvement of Sound Quality of Machinery Noise, J. Acoustical Society of Japan, (in Japanese), Vol.53, No.6 (1997), pp.450-455.

( 3 ) Kuwano, S., Psychological Evaluation of Sound Quality of Machinery Noise, J. Acoustical Society of Japan, (in Japanese), Vol.53, No.6 (1997), pp.456-461.

( 4 ) Kimura, Y., Tanaka, T., Nakajima, H. and Watanabe, H., Improvement of Sound Quality of Engine Exhaust Noise of Hydraulic Excavators, Proc. 3rd Symposium on Environmental Engineering, (in Japanese), No.93039 (1993), pp.21-23.

( 5 ) Kawashima, T., Study for Comfortable Swinging (Comfortable Swinging for Active Rocking Chair), Trans. Jpn. Soc. Mech. Eng., (in Japanese), Vol.69, No.677, C (2003), pp.219-226.

( 6 ) Sayers, B. McA., Analysis of Heart Rate Variability, Ergonomics, Vol.16, No.1 (1973), pp.17-32.

( 7 ) Chess, G.F., Tam, R.M.K. and Calaresu, F.R., Influence of Cardiac Neural Inputs on Rhythmic Variations of Heart Period in the Cat, American Journal of Physiology, Vol.228, No.3 (1975), pp.775-780.

( 8 ) Akselrod, S., Gordon, D., Ubel, F.A., Shannon, D.C., Barger, A.C. and Cohen, R.J., Power Spectrum Analysis of Heart Rate Fluctuation: A Quantitative Probe of Beat-to-Beat Cardiovascular Control, Science, Vol.213 (1981), pp.220-222. 